\title{
Knee chondral lesions treated with autologous chondrocyte transplantation in a tridimensional matrix: clinical evaluation at 1-year follow-up
}

\author{
Félix Vilchez $\cdot$ Jorge Lara $\cdot$ Eduardo Álvarez-Lozano • \\ Carlos E. Cuervo - Oscar F. Mendoza • \\ Carlos A. Acosta-Olivo
}

Received: 11 April 2009/Accepted: 28 September 2009/Published online: 20 November 2009

(C) Springer-Verlag 2009

\begin{abstract}
Background Despite the many studies on chondral injury repair, no outcomes have been evaluated with the Western Ontario and McMaster (WOMAC) Universities osteoarthritis index, the Knee Injury and Osteoarthritis Outcome Score (KOOS), and the Oxford Knee Score, all of which are specific for evaluating the presence of osteoarthritis.

Materials and methods We evaluated the clinical progress of patients following autologous chondrocyte implantation (ACI) performed by our Bone and Tissue Bank using a technique in which cells, instead of being introduced to the articular defect in a liquid form, are implanted into a tridimensional matrix of semisolid collagen $\left(\right.$ Condrograft $\left.{ }^{\circledR}\right)$. A total of 22 patients underwent the procedure, 15 of whom were available for a 1-year followup that included clinical evaluation by WOMAC score before and after surgery and KOOS and the Oxford Knee Score after surgery.

Results The results were improved WOMAC score from 56.4 before surgery to 16.2 after surgery $(P<0.002)$, average KOOS score of 83.6, and average Oxford Knee Score of 18.8 .
\end{abstract}

F. Vilchez · C. E. Cuervo - O. F. Mendoza

C. A. Acosta-Olivo ( $\square)$

4to Piso Depto Ortopedia y Traumatología,

Hospital Universitario "Dr. José E. González"

Ave. Madero y Gonzalitos s/n,

Mitras Centro, CP 64480 Monterrey, NL, Mexico

e-mail: acostaolivocarlos@gmail.com

J. Lara · E. Álvarez-Lozano

Bone and Tissue Bank,

University Hospital "Dr. José E. González",

Monterrey, Mexico

e-mail: dedaloz@bancodehueso.org
Conclusions These results indicate that our tridimensional matrix technique effectively improved patients' quality of life, at least in the short term, and delayed any subsequent procedure. Long-term assessment is necessary to determine the true value of this technique.

Keywords Tridimensional matrix - WOMAC score . Clinical evaluation · Oxford Knee Score · KOOS

\section{Introduction}

Injuries to the articular cartilage are complex and difficult to repair, due mainly to the poor capacity of this tissue to repair itself, which produces a scar tissue with histological and biomechanical characteristics inferior to the original cartilage. This can create failures in the injured joint, which can cause arthritis [1]. Different repair techniques, such as microfractures, mosaicplasty, and osteochondral allograft, among others, have attained positive results [2-6]. Brittberg et al. [2], in 1994, reported autologous chondrocyte implantation (ACI) as being an effective technique for repairing articular defects. The original technique required injecting cells into the chondral defect, and these cells were covered with a periosteal patch that, due to its chondrogenic potential, allowed tissue repair with histological characteristics similar to the hyaline cartilage $[2,7,8]$. This technique requires more time in surgery, and complications such as hypertrophy and delamination have been reported with the use of periosteum [6, 8-11].

In our Bone and Tissue Bank, we are attempting to improve the ACI method by using a technique in which cells, instead of being introduced to the articular defect in a liquid form, are implanted into a tridimensional matrix of semisolid collagen $\left(\right.$ Condrograft $\left.{ }^{\circledR}\right)$. In this way, the graft is 
obtained more easily, completely covers the injury, does not require the periosteum patch, and requires less surgical time. In this paper, we report our clinical assessment of patients treated with this tridimensional matrix, using specific scores for patients with osteoarthritis [Western Ontario and McMaster (WOMAC) Universities osteoarthritis index, the Knee Injury and Osteoarthritis Outcome Score (KOOS) and the Oxford Knee Score], because we found no records that use this type of evaluation.

\section{Materials and methods}

We considered all patients who underwent an ACI, and whose cells were processed in our Bone and Tissue Bank, between January 2005 and September 2007. A total of 22 patients underwent ACI; seven were excluded, of whom three were subjected to implant in talus bone and the remaining patients either did not respond or did not agreed to the interview. We therefore followed 15 patients, of whom six were men and nine were women, with a mean age of 42.5 (29-54) years.

The inclusion criteria were knee pathology, chondral defects demonstrated by magnetic resonance imaging (MRI) and/or arthroscopy, injuries of $1.5-8 \mathrm{~cm}^{2}$, no knee instability (anterior, posterior, or lateral), and no injuries on "mirror." Exclusion criteria were having had surgery in a previously injured area, preliminary procedures for treating chondral injuries, osteoarthritis (OA) of two or more knee compartments, and refusal of the proposal and evaluation technique. Patients who did not complete the assessment or who were without an effective follow-up were eliminated. All patients provided written informed consent to participate.

All patients underwent arthroscopic surgery to obtain a biopsy of autologous chondrocyte. The cells were then processed through the standard technique [12], and when the cellular count was considered high enough, cells were placed in a tridimensional matrix (Condrograft ${ }^{\circledR}$ ) and the implantation performed using a minimum arthrotomy, lateral or medial, depending on injury site. The matrix was fixed by sutures through drill holes in the bone, and the procedure was performed by placing cuff ischemia in the upper third of the thigh. The immediate postoperative protocol was similar for all patients; they were sent home between 1 and 2 days after surgery, with no weight bearing permitted for 4 weeks to enable proper adherence of the patch. After this period, the criterion for rehabilitation was carried out by the physician in charge of the patients and their rehabilitation team. According to this criterion, patients were allowed to move on demand as flexibility increased.

All patients were evaluated using the WOMAC score before and after surgery, and the Oxford Knee Score and KOOS after surgery. Postsurgical evaluation was carried out a year after surgery. The WOMAC score evaluates knee OA, but not appearance or function, ability to perform sports, or quality of life. The KOOS, as an extension of the WOMAC score, allows evaluation of young patients with knee injury over the short and long term on five subscales: Pain, Other Symptoms, Daily Living Activities, Sport and Recreation Function, and Quality of Life. The Oxford Knee Score consists of 12 questions and assesses good function or the need for further treatment [13-17]. Our study was approved by the ethical committee of our hospital, in accordance with the Declaration of Helsinki.

Statistical analysis

We conducted the Wilcoxon signed ranks test and the sign test, with correlated data with a reliability of $95 \%$. Evaluation included the WOMAC score before and after surgery, as well as analysis by gender and affected side. Significance was set at $P<0.05$, and the statistics program STATA-IC-10 was used for statistical analysis.

\section{Results}

Of the 15 patients studied, six were men and nine were women, with an age average of 42.5 (range 29-54) years. As for the side of the injury, seven were left and eight were right. Regarding lesion location, nine were in the lateral condyle, four were medial, one was a patella lesion, and one involved both condyles (Table 1).

WOMAC score before surgical procedure

WOMAC score before surgery was 56.4 points (22-96). Grouped by gender, men scored 52.5 (28-81) and women 59 (22-96). Patients with a left-sided injury scored 59.8 (28-96) before surgery, whereas those with a right-sided injury scored 51.5 (22-87). According to injury location, patients with an injured lateral condyle scored a mean of 46.2 (22-87), whereas those with medial-side injury scored 69.2 (51-96). The patient injured in both condyles scored 72 , whereas the patient with a patella injury scored 81 (Table 2).

WOMAC score after surgical procedure

All but two patients showed improvement after surgery, with a mean WOMAC score of $16.2(2-74)$. The average score for men was 11.1 (5-23) and for women, 19.7 (2-74). Patients with left-sided injury scored an average of 18.4 (274) and those with right-sided injury 15.1 (4-37). In patients with lateral injury, WOMAC improved to 12.1 (437 ), and patients with medial injury scored a mean of 9.75 
Table 1 Overview of patient data

Mean of patients by sex and total average

WOMAC Western Ontario and McMaster Universities osteoarthritis index, KOOS

Knee Injury and Osteoarthritis Ourtome Score

\begin{tabular}{llll}
\hline & Men & Women & Total average (range) \\
\hline Number of patients & 6 & 9 & \\
Age & $43.1(38-53)$ & $42.1(29-54)$ & $42.5(29-54)$ \\
WOMAC score before surgery & $52.5(28-81)$ & $59(22-96)$ & $56.4(22-96)$ \\
WOMAC score after surgery & $11.1(5-23)$ & $19.7(2-74)$ & $16.2(2-74)$ \\
KOOS & $88.1(79-95)$ & $80.5(41-92)$ & $83.6(41-95)$ \\
Oxford Knee Score & $16.8(14-19)$ & $20.1(15-31)$ & $18.8(14-31)$ \\
\hline
\end{tabular}

Table 2 Data pertaining to injury site

\begin{tabular}{llllll}
\hline & Age & WOMAC before & WOMAC after & KOOS & Oxford Knee Score \\
\hline Medial condyle & $42.2(29-53)$ & $46.2(22-87)$ & $12.1(4-37)$ & $86.7(67-95)$ & $17.5(14-28)$ \\
Lateral condyle $^{\mathrm{a}}$ & $41.5(29-56)$ & $69.2(51-96)$ & $9.7(2-14)$ & $88.2(85-92)$ & $18.5(17-19)$ \\
Both condyles $^{\mathrm{a}}$ & 54 & 72 & 74 & 41 & 31 \\
Patella $^{\mathrm{a}}$ & 38 & 81 & 23 & 79 & 19 \\
\hline
\end{tabular}

WOMAC Western Ontario and McMaster Universities osteoarthritis index, KOOS Knee Injury and Osteoarthritis Outcome Score

${ }^{a}$ In both cases, single-patient data

(2-14). The patient with a lesion on both condyles showed a worsened score after surgery (74), whereas the score for the patient with patella injury improved to 23 (Table 2).

\section{Oxford Knee Score}

The average Oxford Knee Score was 18.8 (14-31), which indicates a satisfactory joint function for all patients. Men had an average score of 16.8 (14-19), whereas women averaged 20.1 (15-31). Patients with left-sided injuries scored an average of 18.5 (14-31) and those with rightsided injuries an average of 19 (15-28). In general, in all groups, a score $<20$ was obtained. Patients with lateral condyle injury scored an average of 17.5 (14-28) and those with medial injury $18.5(17-19)$. The patient with a lesion on both condyles scored 31 , a level of moderately severe osteoarthritis, whereas the patient with patella injury scored 19 (Table 2).

\section{KOOS}

The average KOOS was 83.6 (41-95). Men scored an average of 88.1 (79-95), whereas women scored an average of 80.5 (41-92). Patients with left-sided injury scored an average of 82.4 (41-95), whereas those with right-sided injury scored an average of 83.9 (67-92). Patients with lateral condyle injury scored a mean of 86.7 (67-95), and those with medial condyle injury scored 88.2 (85.1-92.2). Patients with both condyles injured scored 41.07 , whereas patients with an injured patella scored 79.16 (Table 2).

\section{Comparison of WOMAC scores}

WOMAC score before surgery was 56.4 points and after surgery $16.33(P<0.002$ on Wilcoxon signed ranks test and $P<0.007$ on Sign test), which was a statistically significant difference. Women before surgery scored 59 points and after surgery $19.7(P<0.0033)$; men before surgery scored 52.5 points and after surgery 11.1 points $(P<0.0001)$. Patients affected on the left side scored 60 points before surgery and 18.4 points after surgery $(P<0.0025)$. Result for the right side before surgery was 53 points and after surgery 15.1 points $(P<0.0002)$ (Table 2). These results showed a statistically significant difference.

\section{Discussion}

There have been reports about treatment of chondral injuries with bilayer devices of collagen compared with pig collagen type I/III in which the same results were reported with both techniques [18]. Other alternatives have been described, such as reabsorbable membrane Chondroidglide (collagen type I/III membrane), and clinical outcomes with this type of membrane seem promising $[19,20]$. There have been reports of good short-term results using a scaffold biodegradable polymer-based hyaluronan HYAff-11 [21]. We believe that our tridimensional matrix is just as effective as other implants, as we create a semisolid environment that allowed cells to better establish and be 
properly maintained at the injury site. In addition, our clinical results in the short term are promising.

We chose the Oxford, WOMAC, and KOOS, as these methods indicate development of OA and have proven their reliability in assessing and evaluating such patients. KOOS is an extension of WOMAC [13] and was developed for the evaluation of young patients with knee injury or OA in the short- and long term. It consists of 42 items separated into five subscales: Pain, Other Symptoms, Daily Living Activities, Sports and Recreation Function, and Quality of Life. There are five possible answers in each subscale with a value from 0 (no problem) to 4 (extreme problem) ranked on a scale of $0-100$, where 0 represents extreme knee problems and 100 trouble free $[14,15]$. Two advantages with KOOS compared with WOMAC are the subscale of Sports and Recreation Function, as well as Quality of Life, because patients with OA subjected to procedures for their improvement, manifested a significant breakthrough in these parameters [14].

After a year of evaluation, we found a significant improvement of symptoms in the WOMAC score. Patients obtained 56.4 points before surgery and 16.2 points a year after the procedure. Bentley et al. [22], in 2003, compared the use of mosaicplasty with ACI and found good and excellent results in $88 \%$ of patients treated with ACI and $69 \%$ in patients treated with mosaicplasty using the Cincinnati Score and Stanmore Score after a year of assessment.

Mithöfer et al. [23] conducted a study in soccer players in whom ACI was performed and found a $72 \%$ good to excellent results with a Tegner Activity Score, plus 33\% of their patients returned to physical activity. In our results, the Oxford score was 18.8 points, which is considered within normal limits, without details of OA, whereas with KOOS, the result was 83.6 points out of a possible 100 , which placed our patients in a very acceptable position according to assessment by that score. In addition, Krishnan et al. [24] found improvement in the Cincinnati Score from 46.1 to 68.4 in patients treated with ACI, with good to excellent results in $82 \%$ of patients with juvenile onset of osteochondritis dissecans compared with $44.4 \%$ of those with adult onset of disease. Bartlett et al. [18], in a preliminary report, valued the ACI with a bilayer membrane of collagen, modifying the original technique of "sandwich," avoiding the use of periosteum. They evaluated patients with a modified Cincinnati Score, analogue scale for pain, and functional Stanmore Score. All patients improved after 6 months and even further after a year. They ranked good to excellent after 6 months. Wood et al. [25] reported 497 adverse effects in 294 patients receiving Carticel, of whom $96 \%$ of had femoral condyle injuries. The most reported side effect was graft failure in $25 \%$, delamination in $22 \%$, and implant hypertrophy $18 \%$. There were also 18 infections reported: seven soft tissue and 11 joint infections; in general, there were adverse effects in $3.8 \%$ of patients treated with this method between 1995 and 2002.

Among the limitations of our study was the need to conduct a long-term assessment, complemented with clinical results of MRI images, as well as the lack of a secondlook arthroscopy to assess membrane integration. These data would complement the clinical assessment. We concluded that ACI with our tridimensional membrane $\left(\right.$ Condrograft $\left.{ }^{\circledR}\right)$ is a good treatment option for patients with osteochondral injuries that meet certain criteria. In addition to the clinical results obtained by our study, patients improved significantly after application of this technique.

Acknowledgments Special thanks to Roberto Acosta, who helped us in the draft of this document, and to Cesar Luna Gurrola, who helped us with the statistical evaluation.

Conflict of interest statement The authors hereby certify that there is no actual or potential conflict of interest in relation to this article.

\section{References}

1. Mankin HJ (1982) The response of articular cartilage to mechanical injury. J Bone Joint Surg Am 64:460-466

2. Blevins FT, Steadman JR, Rodrigo JJ, Silliman J (1998) Treatment of articular cartilage defects in athletes: an analysis of functional outcome and lesion appearance. Orthopaedics 21:761768

3. Brittberg M, Lindahl A, Nilsson A, Ohlsson C, Sjögren-Jansson E, Peterson L (1994) Treatment of deep cartilage defects in the knee with autologous chondrocyte transplantation. N Eng J Med 331:889-895

4. Chu C, Convery F, Akeson W, Meyers M, Amiel D (1999) Articular cartilage transplantation: clinical results in the knee. Clin Orthop Rel Res 360:159-168

5. Mandelbaum B, Browne J, Fu F, Micheli L, Mosely J Jr, Erggelet $\mathrm{C}$ et al (1998) Articular cartilage lesions of the knee. Am J Sport Med 26:853-861

6. Minas T (1999) The role of cartilage repair techniques, including chondrocyte transplantation in focal chondral knee damages. Instr Course Lect 48:629-643

7. Peterson L, Minas T, Brittberg M, Sjögren-Jansson E, Lidahl A (2000) Two- to 9-year outcome alter autologous chondrocyte transplantation of the knee. Clin Orthop 374:212-234

8. O'Driscoll S, Fitzsimmons J (2001) The role of periosteum in cartilage repair. Clin Orthop 391:190-197

9. Minas T, Peterson L (1999) Advanced techniques in autologous chondrocyte transplantation. Clin Sport Med 18:13-44

10. Micheli L, Browne J, Erggelet C, Fu F, Mandelbaum B, Moseley $\mathrm{J}$ et al (2001) Autologous chondrocyte implantation of the knee: multicenter experience and minimum 3-year follow-up. Clin J Sport Med 264:348-357

11. Ueno T, Kagawa T, Mizukawa N, Nakamura H, Sugahara T, Yamamoto T (2001) Cellular origin of endochondral ossification from grafted periosteum. Anat Rec 264:348-357

12. Jones D, Peterson L (2006) Autologous chondrocyte implantation. J Bone Joint Surg Am 88:2501-2520 
13. Bellamy N, Buchanan W, Goldsmith C, Campbell J, Stitt L (1998) Validation study of WOMAC: a health status instrument for measuring clinically important patient relevant outcomes to antirheumatic drug therapy in patients with osteoarthritis of the hip or knee. J Rheumatol 15:1833-1840

14. Roos E, Toksvig-Larsen S (2003) Knee injury and osteoarthritis outcome score (KOOS) - validation and comparison to the WOMAC in total knee replacement. Health Qual Life Outcomes I:17

15. Roos E, Lohmander S (2003) The knee injury and osteoarthritis outcome score (KOOS): from joint injury to osteoarthritis. Health Qual Life Outcomes I:64

16. Bellamy N, Kirwan J, Boers M, Brooks P, Strand V, Tugwell P et al (1997) Recommendations for a core set of outcome measures for future phase III clinical trials in knee, hip and hand osteoarthritis. Consensus development at OMERACT III. J Rheumatol 24:799-802

17. Brazier J, Harper R, Munro J, Walters S, Snaith M (1999) Generic and condition-specific outcome measures for people with osteoarthritis of the knee. Rheumatology (Oxford) 38:870-877

18. Bartlett W, Skinner J, Gooding C, Carrington R, Flanagan A, Briggs $\mathrm{T}$ et al (2005) Autologous chondrocyte implantation versus matrix-induced autologous chondrocyte implantation for osteochondral defects of the knee. J Bone Joint Surg $\mathrm{Br}$ 87-B:640-645

19. Behrens P, Ehlers E, Kochermann K, Rohwedel J, Russlies M, Plotz W (1999) New therapy procedure for localized cartilage defects. Encouraging results with autologous chondrocyte implantation. MMW Fortschr Med 141:49-51

20. Russlies M, Behrens P, Wunsch L, Gille J, Ehlers E (2002) A cellseeded biocomposite for cartilage repair. Ann Anat 184:317-323

21. Grigolo B, Lisignoli G, Piacentini A, Fiorini M, Gobbi P, Mazzotti G et al (2002) Evidence for redifferentiation of human chondrocytes grown on a hyaluronan-based biomaterial (HYAff 11): molecular, immunohistochemical and ultrastructural analysis. Biomaterials 23:1187-1195

22. Bentley G, Biant L, Carrington R, Akmal M, Goldberg A, Williams A et al (2003) A prospective, randomised comparison of autologous chondrocyte implantation versus mosaicplasty for osteochondral defects in the knee. J Bone Joint Surg $\mathrm{Br}$ 85-B:223-230

23. Mithöfer K, Peterson L, Mandelbaum B, Minas T (2005) Articular cartilage repair in soccer players with autologous chondrocyte transplantation. Functional outcome and return to competition. Am J Sport Med 33(11):1-8

24. Krishnan S, Skinner J, Carrington R, Flanagan A, Briggs T, Bentley G (2006) Collagen-covered autologous chondrocyte implantation for osteochondritis dissecans of the knee. J Bone Joint Surg Br 88-B:203-205

25. Wood J, Malek M, Frassica F, Polder J, Mohan A, Bloom E et al (2006) Autologous cultured chondrocyte: adverse events reported to the United States Food and Drug Administration. J Bone Joint Surg Am 88:503-507 